\title{
Respiratory monitoring with electrical impedance tomography during Alveolar recruitment maneuver in a patient with impairment of respiratory function
}

\author{
Antonio Romero, Javier García. Department of Anesthesiology, Resuscitation and Critical Care. \\ Hospital Universitario Puerta de Hierro-Majadahonda , Madrid, Spain.
}

\section{Introduction}

One problem during hospitalization of patients is the impairment of respiratory function and hemodynamic instability.

Sometimes is difficult to transfer the patient to perform a chest Computed Tomography(CT) because of their hemodynamic status. The lungs undergoing mechanical ventilation may have areas with atelectasis, which favors inhomogeneus Tidal

Volume(Tv)distribution. This lack of homogeneity has been studied by CT but do not allow monitoring bedside. Electrical Impedance Tomography(EIT) is free of radiation and has a high resolution, suitable for monitoring the Tv distribution in the lung through the electrical impedance during each breathing cycle immediately helping us in selecting ventilator settings.

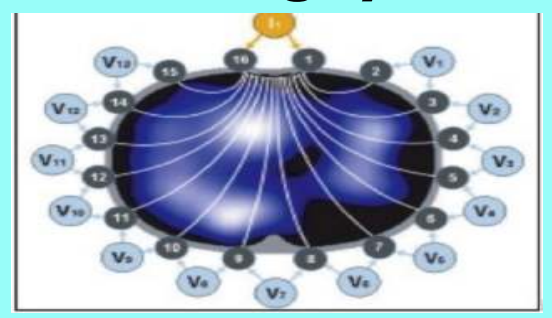

More air contains the lung, higher will be the resistance to the step of the electrical unload. To determine this impedance a belt with 16 electrodes( 2 of them apply a low current, and 14 measuring the resulting tensions) is incorporated around the patient's chest that are distributed around the chest. The resulting image corresponds to the rib section $360^{\circ}$ surrounding the electrodes. Blue image representing ventilated area, white area reaches the highest \% of Tv and black unventilated regions.

\section{Case Presentation}

Male patient with cardiogenic shock who was implanted a biventricular cardiac assistance. During the stay in the critical care unit suffered a deterioration of respiratory function. Respirator parameters were adjusted to avoid lung distension. The distribution of tidal volume was monitored with EIT, bedside, and most of the Tr was not evenly distributed in the lungs. Most of the volume, $42 \%+29 \%(71 \%)$ was in right lung field and only $17 \%+4 \%(21 \%)$ was distributed to the left lung. (Figure I).

Alveolar recruitment maneuver (ARM) under TIE monitoring allowed to observe the distribution of $\mathrm{Tr}$ and what was the alveolar opening pressure needed to maintain homogeneously ventilated the lung. During AMR we observed improvement in the distribution of Tv.

EIT showed with the Tv distribution was more homogeneous, deriving

$52 \%$ to the right lung and $37 \%$ to the left lung. The maximum compliance(Dc) achieved was $37 \mathrm{ml} / \mathrm{hPa}$ to decrease PEEP below 8 $\mathrm{cmH} 2 \mathrm{O}$ during the expiratory phase of the AMR and this correlated with the image of the EIT in which \% of ventilated left lung field decreases.

We established as optimal PEEP $10 \mathrm{cmH} 2 \mathrm{O}, 2 \mathrm{cmH} 2 \mathrm{O}$ over the PEEP under which we looked the collapse with EIT and the fall of the Dc.Tv distribution was more homogeneous, with improved ventilation of the left lung. (Figure 2,3).

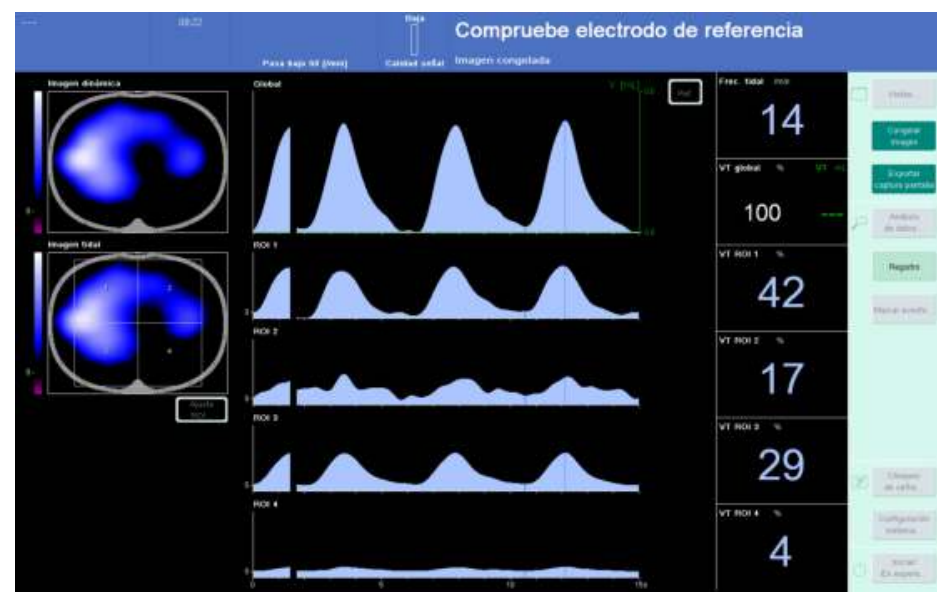

FIGURE I:

$71 \%$ of $T v$ is in right lung

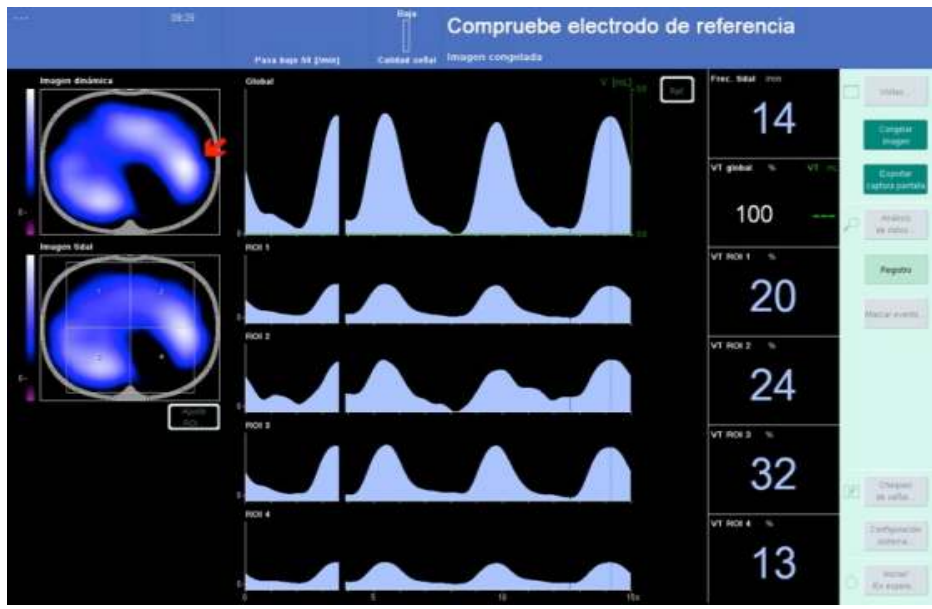

FIGURE 2

After MRA, Tv distribution was more homogeneus
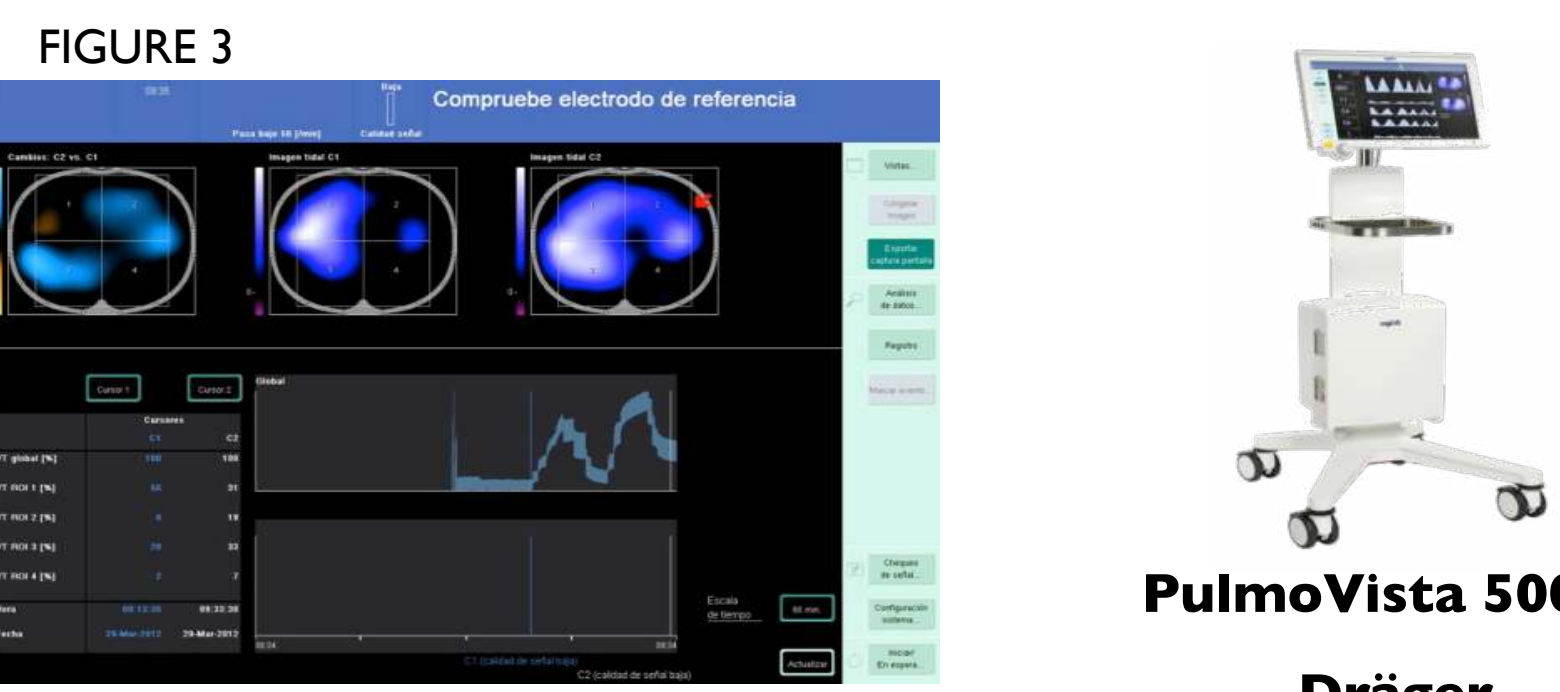

PulmoVista 500

\section{Dräger}

Discussion

Mechanical ventilation can cause damage. That concept has been called lung injury induced by mechanical ventilation (VILI).

The VILI relates to the application of a insufficient PEEP level to prevent alveolar collapse (atelectrauma) and to use high alveolar pressures

(barotrauma). Also the high tidal volume (volutrauma) seems able to induce cyclical alveolar distension.

Preventing alveolar overdistension can be achieved using $T v \leq 6 \mathrm{ml} / \mathrm{kg}$ ideal weight and Pplateau $<30 \mathrm{cmH} 2 \mathrm{O}$.

EIT lets see directly the effectiveness of the ARM, and therefore helps to set the PEEP level.

EIT has proven to be a technique that can monitor changes in lung volume during mechanical ventilation bedside'.

In this case EIT identified that Tr not evenly distributed throughout the lung fields, before making the ARM, and allowed to observe directly the gain lung volume ventilated during ARM guiding until pressure we can come during the MRA

\section{Conclusions}

EIT bedside could be an alternative to CT scans in evaluating the ventilation of lung regions 1 .

EIT is a useful clinical monitor, which in our case allowed to observe, bedside, distribution of tidal volume and monitoring the effectiveness of the MRA, thereby optimizing the adjustment of ventilator settings. 